\title{
Influência do aleitamento materno na depressão pós-parto: revisão sistematizada
}

\author{
Influence of breastfeeding on postpartum depression: systematic review
}

\author{
Karina Rodrigues Santana ${ }^{a}$ \\ Orcid: https://orcid.org/0000-0003-4672-7451 \\ Denise Leite Maia Monteirob \\ Orcid: https://orcid.org/0000-0003-4679-1859 \\ Leila Cristina Soares ${ }^{\mathrm{c}}$ \\ Orcid: https://orcid.org/0000-0001-8360-3189
}

\author{
Nádia Cristina Pinheiro Rodrigues ${ }^{\mathrm{d}}$ \\ Orcid: https://orcid.org/0000-0002-2613-5283
}

\author{
Roberta Monteiro Raupp ${ }^{\mathrm{e}}$ \\ Orcid: https://orcid.org/0000-0001-9634-8850
}

Abilene do Nascimento Gouvêa
Orcid: https://orcid.org/0000-0002-3033-5069

\begin{abstract}
Resumo
Introdução: A depressão é a terceira causa de morbidade do mundo e acredita-se que possa ser a primeira em 2030. O período gravídico-puerperal é o de mais alto risco, pois envolve fatores hormonais, físicos e emocionais. No Brasil, a depressão pós-parto (DPP) afeta 13-19\% das mães no período até 6 meses pós-parto, correspondendo ao tempo de aleitamento exclusivo preconizado pela Organização Mundial da Saúde. A amamentação exclusiva traz muitos benefícios para mãe e filho, no entanto, fatores psicológicos estão envolvidos na sua iniciação e duração. Objetivo: Conhecer a associação entre amamentação e DPP. Método: Revisão sistematizada a partir de pesquisa nas bases de dados eletrônicas: Medline (PubMed), LILACS e SciELO, usando como estratégia de busca: "Postpartum Depression"[Mesh] AND "Breastfeeding"[Mesh], a partir de 2013. Essa revisão incluiu 18 artigos sendo 14 coortes (13 prospectivos e 1 retrospectivo), 3 estudos transversais e 1 ensaio clínico. Resultados: A DPP está associada à gravidez na adolescência, maior peso materno, início tardio do pré-natal, tabagismo, à depressão pré-natal e a fatores pós-parto como $>$ nível de cortisol, < nível de ocitocina, alterações psicológicas e escala de depressão pós-parto de Edimburgo $\geq 10$. Conclusões: Fatores ligados à DPP podem ser identificados já durante o pré-natal ou surgirem no pós-parto. Há associação entre desmame precoce e DPP, porém, não se pode concluir se a cessação da amamentação é consequência ou fator desencadeante da DPP. A detecção precoce desses fatores permite o controle da DPP, podendo evitar a interrupção da amamentação.

Palavras-chave: depressão pós-parto; aleitamento materno.
\end{abstract}

\begin{abstract}
Introduction: Depression is the third leading cause of morbidity in the world and is believed that will rank first by 2030. The pregnancy-puerperal cycle is the highest risk, as it involves hormonal, physical and emotional factors. In Brazil, postpartum depression (PPD) affects 13-
\end{abstract}

\footnotetext{
a Graduanda em Medicina. Centro Universitário Serra dos Órgãos (UNIFESO). E-mail: rodrigues@yahoo.com.br

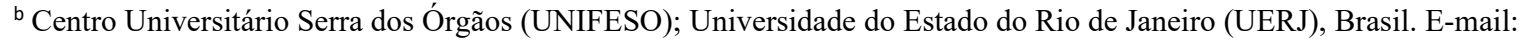
denimonteiro2@yahoo.com.br

${ }^{c}$ Universidade do Estado do Rio de Janeiro, Brasil. E-mail: 1cs1507@yahoo.com.br

${ }^{d}$ Universidade do Estado do Rio de Janeiro (UERJ); Escola Nacional de Saúde Pública (ENSP), Brasil. E-mail: nadiacristinapr@gmail.com

e Pesquisadora associada: Laboratório de Informação Científica e Tecnológica em Saúde. Pós-doutoranda em Informação e Comunicação em Saúde. Instituto de Comunicação e Informação Científica e Tecnológica em Saúde/ Fundação Oswaldo Cruz (ICICT/ FIOCRUZ), Brasil. E-mail: robertamont@gmail.com

${ }^{f}$ Doutoranda em Ciências pela PGCM-UERJ. Universidade do Estado do Rio de Janeiro, Brasil. E-mail: abilenegouvea@uol.com.br
} 
$19 \%$ of mothers in the period up to 6 months postpartum, corresponding to the exclusive breastfeeding time recommended by the World Health Organization. Exclusive breastfeeding brings many benefits to mother and child however, psychological factors are involved in its initiation and duration. Objective: To know the association between breastfeeding and PPD. Method: Systematic review based on research in the electronic databases: Medline (PubMed), LILACS and SciELO, using as search strategy: "Postpartum Depression" [Mesh] AND "Breastfeeding" [Mesh], from 2013. This review included 18 articles, 14 of which were cohorts (13 prospective and 1 retrospective), 3 cross-sectional studies and 1 clinical trial. Results: PPD is associated with teenage pregnancy, higher maternal weight, late onset of prenatal care, smoking, prenatal depression and postpartum factors such as higher cortisol level, lower oxytocin level, psychological changes, and scale of postpartum depression in Edinburgh $\geq 10$. Conclusions: Factors linked to PPD can be identified during prenatal care or appear in the postpartum period. There is an association between early weaning and PPD, however, it is not possible to conclude whether the cessation of breastfeeding is a consequence or triggering factor of PPD. The early detection of these factors allows the control of PPD and can avoid interruption of breastfeeding.

Keywords: postpartum depression; breastfeeding.

\section{Introdução}

A depressão é a terceira causa de morbidade do mundo e acredita-se que pode ser a primeira em 2030. Segundo a literatura, as mulheres apresentam o dobro de risco para depressão do que os homens. O período gravídico-puerperal é o de mais alto risco, pois envolve fatores hormonais, físicos e emocionais ${ }^{1}$. No puerpério, cerca de $40-80 \%$ das mães apresentam distúrbio de humor leve e transitório, entretanto, algumas desenvolvem depressão com sintomas de duração maior que duas semanas ${ }^{2}$.

$\mathrm{O}$ aleitamento materno exclusivo traz benefícios para a mãe e para o bebê. $\mathrm{O}$ leite materno tem a composição nutricional ideal para o bebê, reduz risco de infecções, asma, obesidade, diabetes, morte súbita infantil, leucemia e propicia um bom desenvolvimento da cavidade oral da criança. Já os benefícios para a mãe incluem menor risco de diabetes mellitus tipo 2, prevenção materna de hemorragia pósparto, redução do risco de câncer de mama e ovário, além de aumentar o vínculo mãebebê $\hat{e}^{2,3}$. Devido às várias vantagens do aleitamento materno exclusivo, a Organização das Nações Unidas (ONU) e Fundo das Nações Unidas para a Infância (UNICEF) recomendam o início desde a primeira hora pós-parto até os seis primeiros meses de vida ${ }^{4,5}$.
No Brasil, a prevalência da DPP é maior do que a média mundial $\mathrm{e}$ afeta aproximadamente $13-19 \%$ das mães seis meses após o parto. É nesse período em que ocorre a sua maior intensidade, ou seja, no mesmo intervalo de tempo que é preconizada a amamentação exclusiva pela $\mathrm{OMS}^{5,6}$.

Mesmo com esses benefícios, a cessação precoce do aleitamento materno exclusivo permanece preocupante para a saúde pública $^{3,6}$. Fatores psicológicos estão envolvidos na sua iniciação e duração. Mulheres com sintomas depressivos poderiam ter menos disposição para manter a amamentação ${ }^{6}$. Além disso, a interrupção do aleitamento favorece o aumento nos níveis de ansiedade e depressão materna ${ }^{3}$.

A depressão pós-parto e a interrupção da amamentação podem estar relacionadas com o sistema neuroendócrino. A ocitocina plasmática, que é liberada durante o trabalho de parto e com a sucção do bebê, apresenta propriedades ansiolíticas, regula o estresse e seus baixos níveis estão associados a sintomas depressivos ${ }^{7}$. Com isso, alguns estudos consideram que a amamentação tem efeito protetor na depressão pós-parto ${ }^{2}$.

Revisão sistemática sugere que a depressão pós-parto cria um ambiente não propício para o desenvolvimento pessoal das mães ou para o desenvolvimento ideal da criança. Parece importante, portanto, 
detectar e tratar a depressão durante o período pós-natal, o mais cedo possível para evitar consequências danosas à saúde materno-infantil ${ }^{8}$. Dada a evidência sobre a importância do tema, esta revisão tem como objetivo conhecer a associação entre a amamentação e a DPP.

\section{Método}

Para realizar esta revisão sistematizada foi realizada ampla pesquisa na literatura médica utilizando as bases de dados eletrônicas: Medline (PubMed), LILACS e SciELO, sendo usada a seguinte estratégia de busca: ("Postpartum Depression"[Mesh]) AND "Breastfeeding"[Mesh]. A pesquisa foi finalizada em fevereiro de 2020 e incluiu artigos publicados a partir de 2013. Foram incluídos artigos que abordassem puérperas lactantes e mulheres com depressão pósparto. Foram excluídos artigos que abordassem antidepressivos que interferissem na saúde do lactente, que envolvessem aspectos culturais da amamentação e programas sociais, assim como os que abordassem o peso materno como fator relacionado a não amamentação.

Os estudos foram divididos entre os dois pesquisadores para extração de dados. Em caso de dúvida, os dois discutiram o artigo para chegar a um consenso. Os estudos foram selecionados de acordo com um formulário de extração de dados padronizado, sendo anotados: autores, ano de publicação, país de origem, objetivo de estudo, tipo de população, tamanho da amostra, desenho do estudo, ferramentas usadas para avaliar DPP materna, prevalência relatada de DPP materna, resultados e conclusão.

No Pubmed foram encontrados 74 artigos e nas outras bases de dados foram encontrados mais 39 artigos, totalizando 113 artigos. Após remoção de 11 duplicações e leitura de títulos e resumos, restaram 73 artigos e, obedecendo-se os critérios de inclusão e exclusão préestabelecidos, foram eliminados mais 46 artigos, restando 27 artigos analisados pelo texto completo (Figura 1).

A análise e síntese dos resultados foram descritos em uma tabela, sendo identificados fatores relacionados à depressão materna que podem ser identificados já durante a gravidez e fatores relacionados à depressão pós-parto. $\mathrm{O}$ nível de evidência dos artigos selecionados foi avaliado utilizando-se a classificação de Oxford $^{9}$. (Tabela 1)

\section{Resultados}

Dos artigos selecionados, 18 estudos (13 coortes prospectivos, 1 coorte retrospectivo, 3 estudos transversais e 1 ensaio clínico) foram incluídos na análise dos resultados desta revisão sistematizada (Tabela 1). Esses resultados foram divididos entre fatores que podem ser identificados durante a assistência pré-natal e fatores associados à depressão pós-parto.

\section{Fatores relacionados à depressão que podem ser identificados durante a assistência pré-natal}

Dentre os fatores que podem ser identificados como de risco nas consultas de pré-natal, Lara-Cinisomo et al. ${ }^{7}$, HahnHolbrook et al. ${ }^{10}$ e Cato et al. ${ }^{11}$ relatam a depressão pré-natal. Os três estudos tratavam-se de coorte prospectivo. LaraCinisomo et $a l^{7}$ acompanharam as pacientes por somente 8 semanas e com um número de participantes muito pequeno (34 mulheres) além de não ter feito controle de variáveis confundidoras, o que diminui a força do seu trabalho. O coorte de HahnHolbrook et al. ${ }^{10}$ apresentava uma amostra maior (205 mulheres), porém com predomínio muito grande de mulheres brancas e casadas de classe média, o que pode não ser reprodutível para a população geral. O estudo de Cato et al. ${ }^{11}$ contemplava um grande número (1217 mulheres). Figueiredo et al. $^{3}$, em estudo de coorte prospectivo, também associaram a depressão pré-natal negativamente com a amamentação, entretanto, o pequeno 
número de participantes no grupo que não iniciou a amamentação foi uma limitação.

No entanto, Pope et al. ${ }^{12}$, em um estudo transversal com 2848 mulheres entre cinco e sete meses após o parto, concluíram, após análises de regressão logística, que a tentativa e duração da amamentação não foram associadas à depressão pós-parto em cinco e seis meses quando dentro do contexto de outros fatores de risco.

Outros fatores citados como maior risco de não amamentação foram a gravidez na adolescência ${ }^{6,7,10,13}$, receber assistência através do bolsa família ${ }^{6}$, início tardio do pré-natal ${ }^{6}$, maior peso materno ${ }^{14}$, baixa educação e tabagismo durante a gravide ${ }^{13}$.

O estudo de Silva et al. ${ }^{6}$, apesar de incluir grande número de participantes avaliadas durante o primeiro ano pós-parto, possui as limitações de coleta de dados de um estudo não prospectivo. $\mathrm{O}$ estudo de coorte de Woolhouse et al. ${ }^{15}$ foi prospectivo e contava com um grande número de participantes e encontrou o dobro de chance de não amamentação no segundo parto.

\section{Fatores associados à depressão pós-parto}

A depressão pós-parto pode ser causa e/ou consequência da não amamentação. LaraCinisomo, et al. ${ }^{7}$ observaram que a depressão pós-parto foi associada à cessação da amamentação antes de 2 meses e a baixos níveis de ocitocina. Cox et al. ${ }^{16}$ observaram que mulheres lactantes com sintomas de ansiedade e depressão tinham significativamente maiores níveis de cortisol e menores de ocitocina quando comparadas com as assintomáticas. Como mencionado previamente, o estudo de LaraCinisomo et al. ${ }^{7}$ apresentou limitações pelo número restrito de participantes, tempo de acompanhamento e falta de controle de variáveis confundidoras. $\mathrm{O}$ trabalho de $\mathrm{Cox}$ et al. ${ }^{17}$ constava de questionário, aplicado a um número pequeno de mulheres, com o objetivo de verificar sensibilidade e especificidade de detecção de depressão.
Namm et al. ${ }^{4}$ observaram maior risco de depressão pós-parto em mulheres que descontinuaram a amamentação até seis meses. Além disso, observaram chance $26 \%$ maior de depressão pós-parto em mulheres submetidas à cesariana. Silva et $a l^{6}$ também observaram associação entre ausência de amamentação e depressão pósparto. Apesar do grande número de participantes do estudo de Silva et al. ${ }^{6}$ a grande limitação é a de se tratar de estudo transversal.

Alguns artigos selecionados utilizaram a Escala de Depressão Pós-Parto de Edimburg (EPDS), projetada por Cox et al. ${ }^{17}$ para avaliar os sintomas depressivos maternos, que avalia o risco materno de depressão pós-parto ${ }^{17,18}$. É a escala mais utilizada para rastreio da doença no mundo, com especificidade de $78 \%$, a sensibilidade de $86 \%$ e um valor preditivo positivo de $73 \%$ para quem obtém o escore maior que $10^{17,19}$. A escala consiste em um questionário com 10 itens, com pontuações de 0 a 3 , baseadas na intensidade dos sintomas. Os escores têm uma variação de 0 a 30 . O ponto de corte recomendado para a detecção de depressão menor e maior são os escores maiores ou iguais a $10^{7,18,19,20}$. Cortes mais altos demonstram maior especificidade para depressão maior ${ }^{13}$.

Imširagić et $a l^{21}$, em coorte prospectivo, concluíram que a escala de depressão pós-parto de Edimburgo (EPDS) elevada foi associada com menor chance de amamentação. Reifsnider et al. ${ }^{20}$ observaram tendência $(p=0,06)$ a EPDS maior aos seis meses pós-parto nas mulheres que não amamentaram exclusivamente, porém sem significância estatística.

Yussuf et $a l .^{22}$, em coorte prospectivo e Brown et al. ${ }^{14}$ também encontraram correlação entre amamentação e menores scores EPDS. Wolhouse et al. ${ }^{15}$ e Figueiredo et $a .^{3}$ observaram que mulheres com maiores escores de sintomas depressivos no $1^{\circ}$ trimestre tiveram $14 \%$ mais chance de não iniciar ou interromper de modo precoce a aleitamento materno 
exclusivo. O trabalho de Yussuf et al. ${ }^{22}$ acompanhou as mulheres somente durante os 3 meses pós-parto. $\mathrm{O}$ estudo de coorte prospectivo de Woolhouse et al. ${ }^{15}$, além de contar com grande número de participantes, acompanhou as mulheres durante 6 meses.

Borra et al. ${ }^{19}$ observaram que, dentre as mães que não estavam deprimidas durante a gravidez, as que conseguiram amamentar conforme planejamento prévio tiveram menor risco de depressão pós-parto. Entretanto, o viés de amostragem resultante da natureza voluntária da participação na pesquisa pode ter influenciado os resultados. Gregory et al. ${ }^{13}$ associaram a dor na amamentação ao aumento da chance de depressão pós-parto, entretanto o estudo constava primariamente de mulheres brancas e com alto nível de educação.

Ahmed et al. ${ }^{18}$ associaram o monitoramento interativo da amamentação ao aumento do aleitamento, com melhora da depressão entre mulheres que amamentaram. Da mesma forma, Verpe et $a .^{23}$, acompanhando 176 mulheres, observaram diferenças no aleitamento de mulheres com depressão que faziam acompanhamento domiciliar, demonstrando que a duração, exclusividade e intensidade da amamentação podem ser melhoradas com acompanhamento interativo. Contrariamente ao achado de Ahmed et al., Verpe et $a .^{23}$ não evidenciaram melhora da depressão com o aleitamento. Kornfeind et al. concluíram que o estresse relacionado à volta precoce ao trabalho pode se associar à DPP por interferir no aleitamento materno ${ }^{24}$.

\section{Discussão}

Embora a maioria das puérperas inicie o aleitamento materno exclusivo logo após o parto, muitas interrompem precocemente. Woolhouse et al. ${ }^{15}$ observaram que das mulheres que iniciam a amamentação, $25 \%$ não permanecem amamentando aos três meses pós-parto e das que ultrapassam esse período, $40 \%$ interrompem até o sexto mês ${ }^{15}$. Imširagić et $a l .{ }^{21}$ observaram que com 6 a 9 semanas pós-parto, apenas $58,3 \%$ das mulheres amamentaram exclusivamente. Na Malásia, observou-se que aproximadamente $46 \%$ permaneceram em aleitamento materno exclusivo até três meses após o parto. ${ }^{22}$

Vários fatores maternos interferem na amamentação e muitos deles podem ser observados durante o pré-natal. Um desses fatores é a idade materna. Alguns estudos descrevem tendência a menores índices de amamentação no grupo de mães jovens. ${ }^{6,12,15}$ Silva et al. $^{6}$ analisaram 2.583 binômios mães-crianças, entre 15 dias e três meses no Nordeste brasileiro e verificaram menor porcentagem de aleitamento materno exclusivo quando a idade materna era menor do que 20 anos. Woolhouse et al. ${ }^{15}$ demonstraram na Austrália que as mães entre 18 e 30 anos apresentaram menor tendência a amamentar durante seis meses após o parto do que as entre 30 e 34 anos.

Outros fatores podem estar envolvidos no desmame precoce, como baixa condição socioeconômica, desequilíbrio emocional e baixa escolaridade $^{12,16}$. O nível de educação materna é diretamente proporcional à propensão ao aleitamento. Para Escobar et $a l .^{25}$, em países desenvolvidos, mães mais instruídas amamentam por mais tempo. Já as mães com baixa educação, geralmente iniciam o pré-natal mais tarde, o que, consequentemente, se traduz em menos tempo para obter acesso a informações sobre os benefícios do aleitamento materno. No estudo de Silva et $a l .^{6}$, mães com escolaridade de 4 a 7 anos foram as que menos amamentaram exclusivamente os seus filhos e mães que recebiam assistência do governo federal através do programa Bolsa Família foram as que menos amamentaram. Entretanto, Kummer et al. ${ }^{26}$ constataram que a partir do sexto mês, há inversão e as mães brasileiras que persistem amamentando exclusivamente são as de menor renda.

A partir do sexto mês, quando acaba o aleitamento exclusivo, algumas mães voltam a trabalhar. $\mathrm{Na}$ Croácia, a mãe 
continua recebendo seu salário durante os primeiros seis meses de licença maternidade. Assim, pode amamentar seu filho e ter seu salário mantido durante todo esse período. Desse modo, o fato de ter emprego não interfere na $\mathrm{AME}^{21}$. No caso do Brasil, geralmente as mães que trabalham fora não encontram empecilho à prática da amamentação, desde que tenham condições favoráveis como licença maternidade e horário no trabalho para o aleitamento $^{6}$. Independente do cargo da mãe, o que tem mais influência é a quantidade de horas trabalhadas, pois os maiores índices de desmame estão nas mães que trabalham mais de 20 horas por semana ${ }^{27}$.

Além dos fatores socioeconômicos, a saúde mental materna influencia na prática da amamentação. Para Brown et $a l{ }^{14}$ as mulheres extrovertidas, estáveis emocionalmente e conscientes têm maior tendência a iniciar e manter a amamentação por mais tempo. Uma possível explicação seria que as mães introvertidas ou ansiosas tenham dificuldade em buscar apoio e desafiar as críticas dos outros. Imširagić et $a l .{ }^{21}$ também concluem que a tendência à instabilidade emocional traz impactos negativos para a aleitamento materno exclusivo. Os resultados do estudo de LaraCinisomo et al. ${ }^{7}$ mostraram que a depressão pré-natal pode estar associada à interrupção precoce da amamentação. Além de depressão, mães com ansiedade no pré-natal também têm tendência a ter menor duração do aleitamento materno. Assim sendo, a detecção de sintomas depressivos e de ansiedade, durante esse período, deve receber a atenção devida durante o prénatal, além de apoio adicional ao início do aleitamento ${ }^{15}$.

Segundo o estudo de Silva et al. ${ }^{6}$, alguns fatores do pré-natal contribuíram para menor taxa de aleitamento materno exclusivo como o fato de ter sido realizado no setor público, o início tardio de pré-natal (terceiro trimestre), menos de cinco consultas no total, falta de orientação sobre amamentação, ausência de exame das mamas durantes as consultas e avaliação materna negativa do pré-natal. Esses dados mostram a importância do pré-natal no quesito amamentação. Além disso, fatores como idade, baixas condições socioeconômicas, menor suporte social e instabilidade emocional também estão relacionados com risco maior de depressão pós-parto ${ }^{12}$.

Baixas concentrações de ocitocina podem estar relacionadas à depressão pósparto. A ocitocina plasmática promove a redução da ansiedade e regula a resposta do eixo hipotálamo-hipófise-adrenal ao estresse, ou seja, modera a liberação de cortisol. A desregulação desse eixo é vista em mulheres com depressão ${ }^{7,16}$.

Lara-Cinisomo et al..$^{7}$ obtiveram resultados que mostraram a associação entre depressão, cessação da amamentação e níveis de ocitocina na oitava semana após o parto. No seu estudo com mulheres latinas, avaliaram a liberação desse hormônio, com sua coleta antes, durante e 10 minutos após a mamada (através do seio ou mamadeira). A amamentação exclusiva estimula com mais frequência a lactopoiese, e consequentemente, há mais ocitocina circulante. Mães que amamentavam exclusivamente tinham níveis mais altos de ocitocina do que as que alimentavam seus bebês com o uso de fórmula e, dentre as mães que cessaram o aleitamento, as deprimidas tinham níveis mais baixos de ocitocina plasmática do que as não deprimidas ${ }^{7}$. Para Cox et al. ${ }^{16}$, as mulheres deprimidas têm reatividade desregulada do eixo de estresse hipotalâmico-hipofisário, o que implica numa sinalização anormal de ocitocina. Com essa sinalização anormal, provavelmente o sistema de recompensa fica alterado e essas mulheres estariam mais propensas a mudanças de humor e de comportamento. Isso implicaria em redução da duração da amamentação, sendo uma explicação para a depressão pós-parto levar à cessação precoce da amamentação.

A depressão pós-parto é um dos principais fatores associados à duração da amamentação. Lara-Cinisomo et al. $^{7}$, em 
estudo feito em Chicago, observou que a depressão pós-parto foi associada à cessação da amamentação antes de dois meses em latinas. $\mathrm{Na}$ Coreia, o risco de depressão foi maior nas mulheres que descontinuaram a amamentação até 6 meses e na Malásia, as mães com maiores pontuações na EPDS foram as que não iniciaram ou não mantiveram a amamentação exclusiva durante três meses. De acordo com esses estudos, as mulheres que cessaram precocemente a amamentação apresentaram risco aumentado de desenvolver depressão pós-parto ${ }^{4,22}$.

No entanto, a depressão também pode levar à cessação precoce da amamentação. Observou-se que as mães com maiores escores de depressão no primeiro trimestre tinham mais chances de interromper a amamentação exclusiva e algumas sequer iniciaram. Nas que mantiveram $o$ aleitamento materno exclusivo durante três meses, houve diminuição dos escores de depressão, mostrando que o aleitamento pode ser fator protetor para a depressão pós-parto ${ }^{3,10}$.

A relação entre a duração da amamentação com a depressão pós-parto ainda é complexa por envolver causa e consequência. Os sintomas depressivos podem dificultar a amamentação, o que eleva o risco de interrupção precoce. Entretanto, a cessação precoce pode provocar sintomas depressivos na mãe. Nesse último caso, as dificuldades da amamentação provocam sofrimento materno ${ }^{15}$.

As mulheres param o aleitamento por diversas razões. Os principais fatores são dificuldade na secreção do leite, dor, estilo de vida materno, preocupação com a imagem corporal, constrangimento ao amamentar em público, falta de apoio e a pedido médico. Razões como dificuldade no aleitamento e dor são as que estão diretamente associadas a menores escores na $\mathrm{EPDS}^{13,14}$.

Antes mesmo do parto, algumas mães nutrem o desejo de amamentar seus filhos. No entanto, após o nascimento, nem todas conseguem pôr em prática o aleitamento materno. Isso pode gerar uma frustração e propiciar o surgimento de sintomas depressivos. Um estudo nos EUA e outro na Inglaterra detectaram que mulheres que conseguiram amamentar conforme o planejado tiveram menor risco de depressão pós-parto. $\mathrm{O}$ risco foi maior nas que planejaram, mas não amamentaram. Entre as americanas, essa associação foi significativa no grupo de renda mais alta $^{13,14}$.

Reifsnider et $a l .{ }^{20}$ estudaram a associação entre o tipo de amamentação (exclusiva e não exclusiva) e a depressão pós-parto. Um mês após o parto, entre as mulheres que não amamentaram havia maior pontuação média na EPDS do que as que amamentaram. Entretanto, seis meses após o parto ocorreu o oposto, o que mostra que esses valores podem variar ao longo do período. As mulheres que amamentaram exclusivamente apresentaram menor pontuação, independentemente do tempo de pós-parto, percebendo, então, menor risco para depressão pós-parto ${ }^{20}$.

Pope et al. ${ }^{12}$ acreditam que a falta de aleitamento pode não ser um fator de risco para a depressão pós-parto. Em sua pesquisa, foram analisados também outros fatores como idade, menor renda, maior estresse, menor suporte social, chegando à conclusão de que a ausência do aleitamento por si só não foi significativa para o desenvolvimento de sintomas depressivos. Esses fatores estudados são fatores de risco tanto para a interrupção precoce do aleitamento, quanto para a depressão pósparto $^{6,12,15}$.

As limitações do presente estudo se relacionam ao fato de os artigos abordarem a associação da amamentação com a depressão pós-parto em diferentes períodos do puerpério, o que pode acarretar interpretação duvidosa dos resultados e contradições. Além disso, muitos artigos afirmam que a interrupção da amamentação provoca depressão pós-parto sem, no entanto, considerarem a possibilidade de que estas mulheres também poderiam 
apresentar outros fatores que levassem à depressão pós-parto, o que demonstra a necessidade de novos estudos que comparem a cessação da amamentação com os outros fatores de risco.

\section{Conclusão}

Fatores ligados à DPP podem ser identificados já durante a gravidez ou surgirem no pós-parto. Portanto, o pré-natal é de extrema importância para uma gravidez e um puerpério saudáveis, podendo detectar fatores que poderão interferir na amamentação e na depressão pós-parto, como gravidez na adolescência, maior peso materno, início tardio do pré-natal, tabagismo e depressão pré-natal.

Mães depressivas são mais propensas a interromper precocemente a amamentação, o que confirma a associação entre desmame precoce e DPP. A detecção precoce desses fatores permite o controle da DPP, podendo evitar a interrupção da amamentação. No entanto, como o estudo não concluiu se a cessação da amamentação é consequência ou fator desencadeante da DPP, é necessária a realização de mais estudos para elucidar essa questão.

\section{Referências}

1. Hartmann JM, Mendoza-Sassi RA, Cesar JA. Depressão entre puérperas: prevalência e fatores associados. Cad Saude Publica. 2017;33(9):e00094016.

2. Niwayama R, Nishitani S, Takamura T, Shinohara K, Honda S, Miyamura T et al. Oxytocin Mediates a Calming Effect on Postpartum Mood in Primiparous Mothers. Breastfeed Med. 2017; 12:103-9. doi:10.1089/bfm.2016.0052

3. Figueiredo B, Canário C, Field T. Breastfeeding is negatively affected by prenatal depression and reduces postpartum depression. Psychol Med. 2014; 44(5):927-36. doi:10.1017/S0033291713001530

4. Nam JY, Choi Y, Kim J, Cho KH, Park EC. The synergistic effect of breastfeeding discontinuation and cesarean section delivery on postpartum depression: a nationwide population-based cohort study in Korea. J Affect Disord. 2017; 218:53-8. doi:10.1016/j.jad.2017.04.048

5. World Health Organization, 2001. Expert consultation on the optimal duration of exclusive breastfeeding. World Health Organization, Geneva, Switzerland. Disponível em: https://www.who.int/nutrition/publications/optimal_duration_of_exc_bfeeding_report_en g.pdf

6. Silva CS, Lima MC, Sequeira-de-Andrade LAS, Oliveira JS, Monteiro JS, Lima NMS et al. Association between postpartum depression and the practice of exclusive breastfeeding in the first three months of life. J Pediatr (Rio J). 2017; 93(4):356-64. doi:10.1016/j.jped.2016.08.005

7. Lara-Cinisomo S, Mc Kenney K, Di Florio A, Meltzer-Brody S. Associations between postpartum depression, breastfeeding, and oxytocin levels in latina mothers. Breastfeed Medicine. 2017; 12(7):436-42. doi: 10.1089/bfm.2016.0213

8. Slomian J, Honvo G, Emonts P, Reginster JY, Bruyère O. Consequences of maternal postpartum depression: A systematic review of maternal and infant outcomes. Womens Health (Lond). 2019;15:1745506519844044. doi: 10.1177/1745506519844044

9. Centre for evidence based medicine. Oxford centre for evidence-based medicine 2011 levels of evidence. Disponível em: https://www.cebm.net/wpcontent/uploads/2014/06/CEBM-Levels-of-Evidence-2.1.pdf. Acessado em 05 Out2019

10. Hahn-Holbrook J, Haselton MG, DunkelSchetter C, Glynn LM. Does breastfeeding offer protection against maternal depressive symptomatology? A prospective study from 
pregnancy to 2 years after birth. Arch WomensMent Health. 2013;16(5):411-22. doi:10.1007/s00737-013-0348-9

11. Cato K, Sylvén SM, Georgakis MK, Kollia N, Rubertsson C, Skalkidou A. Antenatal depressive symptoms and early initiation of breastfeeding in association with exclusive breastfeeding six weeks postpartum: a longitudinal population-based study. BMC Pregnancy Childbirth. 2019;19(1):49. doi: 10.1186/s12884-019-2195-9

12. Pope CJ, Mazmanian D, Bédard M, Sharma V. Breastfeeding and postpartum depression: Assessing the influence of breastfeeding intention and other risk factors. J Affect Disord. 2016; 200:45-50. doi:10.1016/j.jad.2016.04.014

13. Gregory EF, Butz AM, Ghazarian SR, Gross SM, Johnson SB. Are unmet breastfeeding expectations associated with maternal depressive symptoms? Acad Pediatr. 2015; 15(3):319-25. doi:10.1016/j.acap.2014.12.003

14. Brown A, Rance J, Bennett P. Understanding the relationship between breastfeeding and postnatal depression: the role of pain and physical difficulties. J AdvNurs. 2016; 72(2):27382. doi:10.1111/jan.12832

15. Woolhouse H, James J, Gartland D, McDonald E, Brown SJ. Maternal depressive symptoms at three months postpartum and breastfeeding rates at six months postpartum: Implications for primary care in a prospective cohort study of primiparous women in Australia. Women Birth. 2016; 29(4):381-7. doi:10.1016/j.wombi.2016.05.008

16. Cox EQ, Stuebe A, Pearson B, Grewen K, Rubinow D, Meltzer-Brody S. Oxytocin and HPA stress axis reactivity in postpartum women. Psychoneuroendocrinology. 2015; 55:164-72. doi:10.1016/j.psyneuen.2015.02.009

17. Cox JL, Holden JM, Sagovsky R. Detection of postnatal depression. Development of the 10-item Edinburgh Postnatal Depression Scale. Br. J. Psychiatry. 1987; 150:782-6.

18. Ahmed AH, Roumani AM, Szucs K, Zhang L, King D. The effect of interactive web-based monitoring on breastfeeding exclusivity, intensity, and duration in healthy, term infants after hospital discharge. J Obstet Gynecol Neonatal Nurs. 2016; 45(2):143-54. doi:10.1016/j.jogn.2015.12.001

19. Borra C, Iacovou M, Sevilla A. New evidence on breastfeeding and postpartum depression: the importance of understanding women's intentions. Matern Child Health J. 2015; 19(4): 897-907.doi:10.1007/s10995-014-1591-z

20. Reifsnider E, Flowers J, Todd M, BeverBabendure J, Moramarco M. The relationship among breastfeeding, postpartum depression, and postpartum weight in Mexican american women. J Obstet Gynecol Neonatal Nurs. 2016; 45(6):760-71. doi:10.1016/j.jogn.2016.05.009

21. Imširagić AS, Begić D, Sarajlić I, Palavra IR, Orban M. Predictors of exclusive breastfeeding 6-9 weeks after delivery: a prospective cohort study. Psychiatr Danub. 2016; 28(4):395-403.

22. Yusuff AS, Tang L, Binns CW, Lee AH. Breastfeeding and postnatal depression: a prospective cohort study in Sabah, Malaysia. J Hum Lact. 2016; 32(2):277-81. doi:10.1177/0890334415620788

23. Verpe H, Kjellevold M, Moe V, Smith L, Vannebo UT, Stormark KM, Søvik ML, Skotheim S. Early postpartum discharge: maternal depression, breastfeeding habits and different follow-up strategies. Scand J Caring Sci. 2019;33(1):85-92. doi: $10.1111 /$ scs. 12604

24. Kornfeind KR, Sipsma HL. Exploring the Link between Maternity Leave and Postpartum Depression. Womens Health Issues. 2018;28(4):321-326. doi: 10.1016/j.whi.2018.03.008

25. Escobar AMU, Ogawa AR, Hiratsuka M, Kawashita MY, Teruya PY, Grisi S et al. Aleitamento materno e condições socioeconômico-culturais: fatores que levam ao 
desmame precoce. Rev. Brasileira de Saúde Materno Infantil. 2002; 2(3): 25361. doi:10.1590/S1519-38292002000300006

26. Kummer SC, Giugliani ER, Susin LO, Folletto JL, Lermen NR, Wu VY et al. Evolução do padrão de aleitamento materno. Rev. Paul Saúde Pública. 2000; 34(2): 143-8. doi.org/10.1590/S0034-89102000000200007.

27. Gielen AC, Faden RR, O'Campo P, Brown CH, Paige DM. Maternal employment during the early postpartum period: effects on initiation and continuation of breast-feeding. Pediatrics. 1991; 87(3):298-305.

\section{Como citar este artigo:}

Santana KR, Monteiro DLM, Soares LC, Rodrigues NCP, Raupp RM, Gouvêa AN. Influência do aleitamento materno na depressão pós-parto: revisão sistematizada. Rev. Aten. Saúde. 2020; 18(64): 110-123. 


\section{Apêndice 1}

Tabela 1. Descrição do desenho de estudo, amostra, objetivo e resultados dos estudos incluídos

\begin{tabular}{|c|c|c|c|c|}
\hline Autor / ano & $\begin{array}{l}\text { Desenho do } \\
\text { estudo }\end{array}$ & Amostra & Objetivo & Resultados \\
\hline $\begin{array}{l}\text { Cato K et al } \\
\qquad(2019) \\
\text { Suécia }^{11}\end{array}$ & $\begin{array}{l}\text { Coorte } \\
\text { prospectivo }\end{array}$ & 1217 우 & $\begin{array}{l}\text { Avaliar o efeito dos sintomas depressivos } \\
\text { pré-natais e o início precoce da } \\
\text { amamentação com a AME. }\end{array}$ & $\begin{array}{l}\text { Sintomas depressivos na gravidez e a iniciação tardia da amamentação } \\
\text { foram associados a } 4 \text { vezes mais chance de interromper a AME seis } \\
\text { semanas após o parto. }\end{array}$ \\
\hline $\begin{array}{l}\text { Verpe } \mathrm{H} \text { et al } \\
\quad(2019) \\
\text { Noruega }^{23}\end{array}$ & $\begin{array}{c}\text { Coorte } \\
\text { prospectivo }\end{array}$ & 176 & $\begin{array}{l}\text { Explorar a influência do } \\
\text { acompanhamento domiciliar na } \\
\text { associação da depressão e amamentação, } \\
\text { com } 6 \text { semanas e } 6 \text { meses após o parto. }\end{array}$ & $\begin{array}{l}\text { Não houve diferença de sintomas depressivos e hábito de amamentar entre } \\
\text { mulheres que receberam acompanhamento padrão ou domiciliar. }\end{array}$ \\
\hline $\begin{array}{l}\text { Kornfeind KR et } \\
\text { al (2018) } \\
\text { EUA }^{24}\end{array}$ & $\begin{array}{l}\text { Coorte } \\
\text { prospectivo }\end{array}$ & $177 q$ & $\begin{array}{l}\text { Explorar a relação entre a duração da } \\
\text { licença maternidade e a depressão pós- } \\
\text { parto. }\end{array}$ & $\begin{array}{l}\text { A licença maternidade } \leqq 12 \text { semanas pode contribuir com adicional risco } \\
\text { de sintomas depressivos pós-parto por interromper a lactação. Cada semana } \\
\text { adicional de licença foi associada a }<\text { chance de apresentar sintomas de DPP } \\
\text { (OR } 0,58 ; \text { IC95\% } 0,40-0,84) \text {. }\end{array}$ \\
\hline $\begin{array}{l}\text { Lara-Cinisomo, et } \\
\text { al. }(2017) \\
\quad \text { EUA }^{7}\end{array}$ & $\begin{array}{l}\text { Coorte } \\
\text { prospectivo }\end{array}$ & $34 q$ & $\begin{array}{l}\text { Investigar associação entre DPP, } \\
\text { ansiedade, amamentação e níveis de } \\
\text { ocitocina. }\end{array}$ & $\begin{array}{l}\text { A ansiedade, DPN e DPP foram associadas à cessação da amamentação } \\
\text { antes de } 2 \text { meses }(p<0.05) \text { A DPP foi relacionada a baixos níveis de OT. }\end{array}$ \\
\hline $\begin{array}{l}\text { Nam JY et al. } \\
\text { (2017) } \\
\text { Coreia }^{4}\end{array}$ & $\begin{array}{l}\text { Coorte } \\
\text { retrospectivo }\end{array}$ & 81447 우 & $\begin{array}{l}\text { Investigar associação da interrupção da } \\
\text { amamentação e de parto cesáreo com a } \\
\text { DPP até } 6 \text { meses. }\end{array}$ & $\begin{array}{l}666(0,82 \%) \text { apresentaram DPP. O risco de DPP foi maior em } q \text { que } \\
\text { descontinuaram a amamentação até } 6 \text { meses }(\mathrm{OR}=3,23, \mathrm{p}<0,001) \text {. A chance } \\
\text { de DPP foi } 26 \% \text { maior nas }+ \text { com cesariana }(\mathrm{OR}=1,26, \mathrm{p}=0,004) \text {. }\end{array}$ \\
\hline $\begin{array}{l}\text { Silva CS et al. } \\
\qquad(2017) \\
\text { Brasil }^{6}\end{array}$ & $\begin{array}{l}\text { Estudo } \\
\text { transversal }\end{array}$ & $\begin{array}{l}2583 \text { pares } \\
\text { mãe-filho }\end{array}$ & $\begin{array}{l}\text { Investigar a associação entre depressão } \\
\text { pós-parto e ocorrência de AME }\end{array}$ & $\begin{array}{l}\text { AME }=50,8 \% . \mathrm{DPP}=11,8 \% \text { das } \text {. } \text {. Ausência de } \mathrm{AME} \text { foi associada à } \mathrm{DPP} \\
(\mathrm{OR}=1,67 ; \mathrm{p}<0,001), \text { a ser adolescente }(\mathrm{OR}=1,89 ; \mathrm{p}<0,001) \text {, a receber } \\
\text { Bolsa Família }(\mathrm{OR}=1,27 ; \mathrm{p}=0,005) \text {, e início de pré-natal tardio }(\mathrm{OR}=2,14 ; \\
\mathrm{p}=0,03) .\end{array}$ \\
\hline $\begin{array}{l}\text { Imširagić AS et } \\
\text { al. (2016) } \\
\text { Croácia }^{21}\end{array}$ & $\begin{array}{l}\text { Coorte } \\
\text { prospectivo }\end{array}$ & $372 q$ & $\begin{array}{l}\text { Investigar a associação de depressão, } \\
\text { estresse pós-traumático e transtorno de } \\
\text { personalidade com AME. }\end{array}$ & $\begin{array}{l}\mathrm{AME}=58,3 \% \text { em } 6 \text { a } 9 \text { semanas pós-parto. Dobra a chance de AME no } 2^{\circ} \\
\text { parto }(\mathrm{OR}=2,12 ; \mathrm{IC} 95 \% 1,10-4,10) \text {. EPDS elevado foi associado com }< \\
\text { chance de } \mathrm{AME}(\mathrm{OR}=0,92 ; \mathrm{IC} 95 \% 0,85-0,99) \text {. }\end{array}$ \\
\hline
\end{tabular}

Nível de evidência 


\section{Reifsnider E et al. Coorte (2016) prospectivo}

EUA $^{20}$

\section{Woolhouse $\mathrm{H}$ et \\ al. (2016)}

Austrália ${ }^{15}$
Estudo

$\begin{array}{cc}(2016) & \text { Estudo } \\ \text { Canadá }^{12} & \text { transversal }\end{array}$

Ahmed AH et al. Ensaio clínico (2016) randomizado

EUA $^{18}$

$\begin{array}{cc}\text { Yusuff AS et al. } & \begin{array}{c}\text { Coorte } \\ \text { (2015) }\end{array} \\ \text { prospectivo }\end{array}$

Malásia 22

Brown A et al.

(2015)

Reino Unido ${ }^{14}$
150 ○

mexicanas

terminar associação entre DPP e o tipo de amamentação (exclusiva, combinada, ausência) em 1 e 6 meses após o parto.

Explorar associações entre sintomas depressivos maternos e a amamentação nos primeiros seis meses pós-parto.

Avaliar a associação entre a amamentação e depressão pós-parto (com base na EPDS) dentro do contexto de outros fatores de risco conhecidos

Determinar se o monitoramento interativo da amamentação baseado na

Web aumentou a frequência da amamentação e, consequentemente,

diminuição dos sintomas de DPP.

Investigar a relação entre a amamentação plena e sintomas depressivos nos 3 meses pós-parto.

Examinar a relação entre as razões da interrupção da amamentação e sintomas depressivos pós-natal.
Aos 6 meses pós-parto, EPDS > nas que não amamentaram exclusivamente $(\mathrm{p}=0,06)$. Em ambos os momentos, diferença significativa no peso materno $(p=0,02)$ entre lactantes (mesmo que não exclusivo) e não lactantes. Aleitamento reduz DPP e peso.

$76 \%$ amamentaram por 3 meses e $61 \%$ por 6 meses. Mães com sintomas depressivos aos 3 meses tiveram taxas mais baixas de amamentação nos seis meses ( $49 \%$ vs. $61 \%$; OR $=0,55$ IC95\% 0,34-0,90). As características associadas com a não amamentação aos 6 meses pós-parto são: mães jovens, baixa educação e tabagismo na gravidez.

$203(7,2 \%)$ das mulheres tinham DPP pela EPDS (score>12), $233(8.3 \%)$ tinham níveis de depressão subclínica (score 10-12). A tentativa de amamentar e a duração não foram associadas com DPP aos 5-7 meses após o parto. Fatores associados com DPP: menor renda, maior estresse, menor suporte social, não ter história de depressão ou de abuso.

Os membros do grupo de intervenção tinham maiores taxas de aleitamento materno exclusivo em 1,2 e 3 meses $(p=0,027, p<0,001$ e $p=0,002$, respectivamente). Até o final do terceiro no mês, $84 \%$ do grupo de intervenção estava amamentando em comparação com $66 \%$ do grupo controle.

$\pm 46 \%$ das $\bigcirc$ permaneceram em AME até 3 meses após o parto. Essas $\uparrow$ tiveram $<$ média na EPDS $(p<0,001)$ em 1 e 3 meses pós-parto do que as que não iniciaram ou não mantiveram a amamentação por 3 meses.

De 502 mães, 217 amamentaram menos de 6 meses. Dessas, o escore médio na EPDS foi de 7,26. Usando 12 como ponto de corte, 32 우

$(14,7 \%)$ tinham DPP. A correlação entre a duração da amamentação e o escore EPDS foi significativa $(\mathrm{p}<0,001)$ 


\begin{tabular}{|c|c|c|c|}
\hline $\begin{array}{l}\text { Gregory EF et al } \\
(2015) \\
\text { EUA }^{13}\end{array}$ & $\begin{array}{c}\text { Coorte } \\
\text { prospectivo }\end{array}$ & 1501 우 & $\begin{array}{l}\text { Avaliar se as expectativas maternas de } \\
\text { aleitamento materno exclusivo foram } \\
\text { associadas à DPP. }\end{array}$ \\
\hline $\begin{array}{l}\text { Cox EQ et al } \\
\qquad(2015) \\
\text { EUA }^{16}\end{array}$ & $\begin{array}{c}\text { Coorte } \\
\text { prospectivo }\end{array}$ & 47 우 & $\begin{array}{c}\text { Testar a associação } \\
\text { entre os níveis de ocitocina durante a } \\
\text { amamentação e a reatividade ao estresse. }\end{array}$ \\
\hline $\begin{array}{l}\text { Borra C et al } \\
\quad(2015) \\
\text { Inglaterra }^{19}\end{array}$ & $\begin{array}{l}\text { Coorte } \\
\text { prospectivo }\end{array}$ & 14.541 & $\begin{array}{l}\text { Identificar o efeito causal da } \\
\text { amamentação na depressão pós-parto. }\end{array}$ \\
\hline $\begin{array}{l}\text { Figueiredo B et al } \\
\text { (2013) } \\
\text { Portugal }^{3}\end{array}$ & $\begin{array}{c}\text { Coorte } \\
\text { prospectivo }\end{array}$ & 145 ㅇ & $\begin{array}{l}\text { Explorar os efeitos do pré-natal e } \\
\text { depressão pós-parto na amamentação; e o } \\
\text { efeito da amamentação sobre DPP. }\end{array}$ \\
\hline $\begin{array}{l}\text { Hahn-Holbrook J } \\
\text { et al (2013) } \\
\text { EUA }^{10}\end{array}$ & $\begin{array}{c}\text { Coorte } \\
\text { prospectivo }\end{array}$ & 205 우 & $\begin{array}{c}\text { Investigar a relação entre amamentação e } \\
\text { DPP. }\end{array}$ \\
\hline
\end{tabular}

A chance de sintomas depressivos é menor nas $q$ que as expectativas de

AME foram atendidas (OR 0,71,IC 95\% 0,52-0,96). Essa associação foi significativa no grupo de renda mais alta $(\mathrm{n}=862$,OR 0,63 , IC 95\% 0,43 a $0,93)$. A dor na amamentação foi associada com aumento da chance de

$$
\text { DPP (OR 1,50, IC 95\% 1,12-2,00). }
$$

Das $q$ que amamentaram, aquelas com sintomas de ansiedade e depressão $(\mathrm{n}=11)$ tinham significativamente $>$ níveis de cortisol $(\mathrm{p}<0,05)$ e níveis mais baixos de OT quando comparados com as assintomáticas.

Entre as mães não deprimidas durante a gravidez, as que conseguiram amamentar conforme o planejado tiveram menor risco de depressão pósparto. O risco foi maior nas que planejaram, mas não amamentaram.

q com maiores escores de depressão no $1^{\circ}$ trimestre tiveram 0,86 vezes mais chance de não iniciar ou interromper de modo precoce a AME (OR 0,86, IC95\% 0,76-0,99). Diminuição nos escores de depressão foi vista, do parto até 3 meses após, em † que mantiveram lactação $(p<0,10)$.

q depressivas no pré-natal desmamaram seus bebês 2,3 meses antes do que as sem tal sintomatologia. As que amamentaram mais frequentemente mostraram maiores declínios dos sintomas durante 3 meses de pós-parto $(\mathrm{p}<0,05)$.

DPN= Depressão pré-natal; DPP= Depressão pós-parto; OT= ocitocina; EPDS - escala de depressão pós-parto de Edimburgo; AME=aleitamento materno exclusivo; Estados Unidos da América. 


\section{Anexo 1}

Fig. 1 Fluxograma de seleção de estudos . Adaptado do fluxograma PRISMA group 2009.

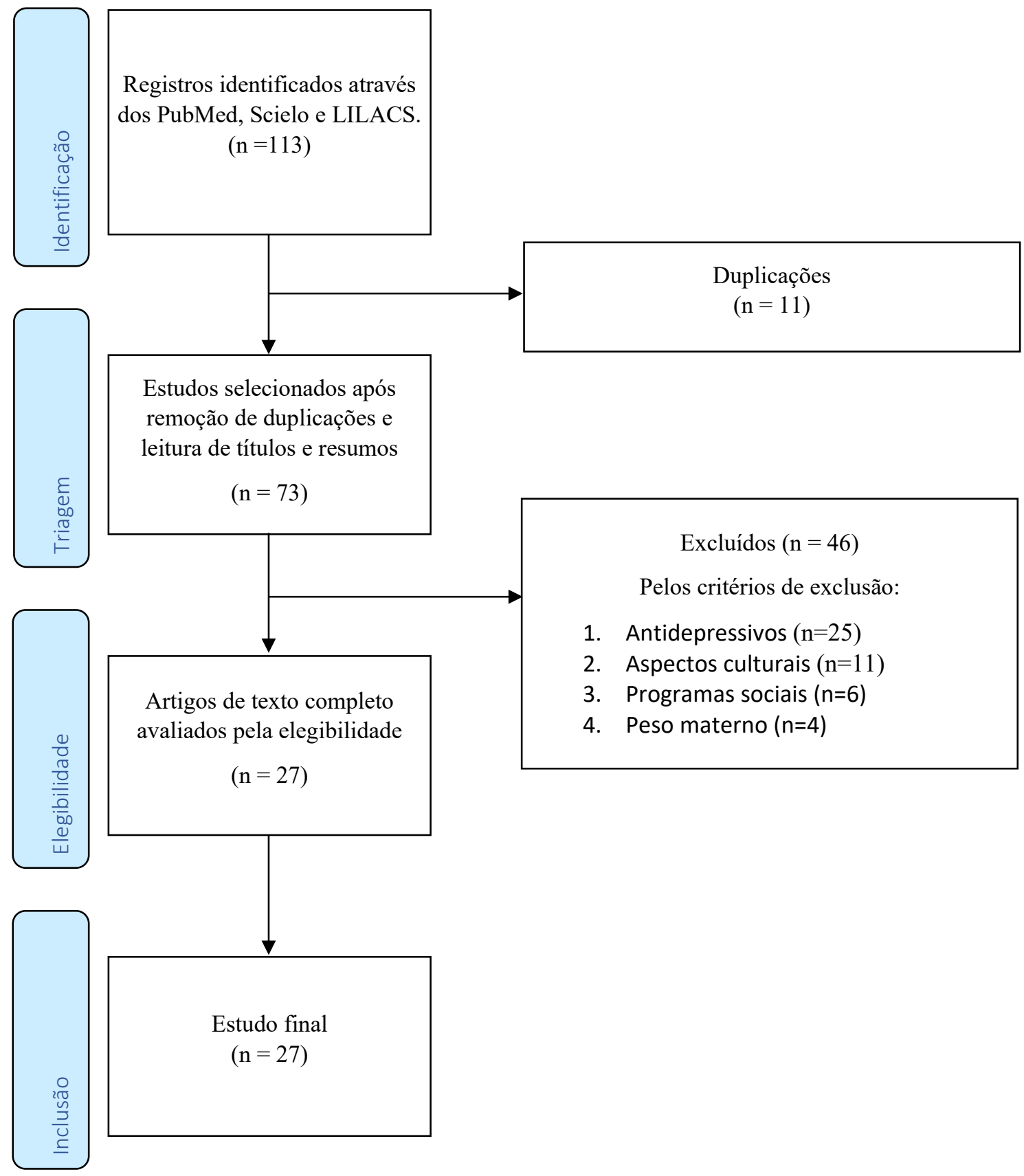

